\title{
Nanoplasmonic Photothermal Heating And Near-Field Enhancements: a Comparative Survey of Nineteen Metals
}

Yael Gutiérrez ${ }^{1,2 *}$, Maria Losurdo², Francisco González ${ }^{1}$, Henry O. Everitt ${ }^{3,4}$ and Fernando Moreno ${ }^{1,2^{*}}$

${ }^{1}$ Optics Group, Department of Applied Physics, University of Cantabria, Avda de Los Castros, s/n 39005 Santander, Spain

2 Institute of Nanotechnology, CNR-NANOTEC, Via Orabona 4, 70126 Bari, Italy

${ }^{3}$ U.S. Army CCDC Aviation \& Missile Center, Redstone Arsenal, Alabama 35898, USA

${ }^{4}$ Department of Physics, Duke University, Durham, North Carolina 27708, USA

\section{Group II A materials}

- $\mathrm{Mg}$
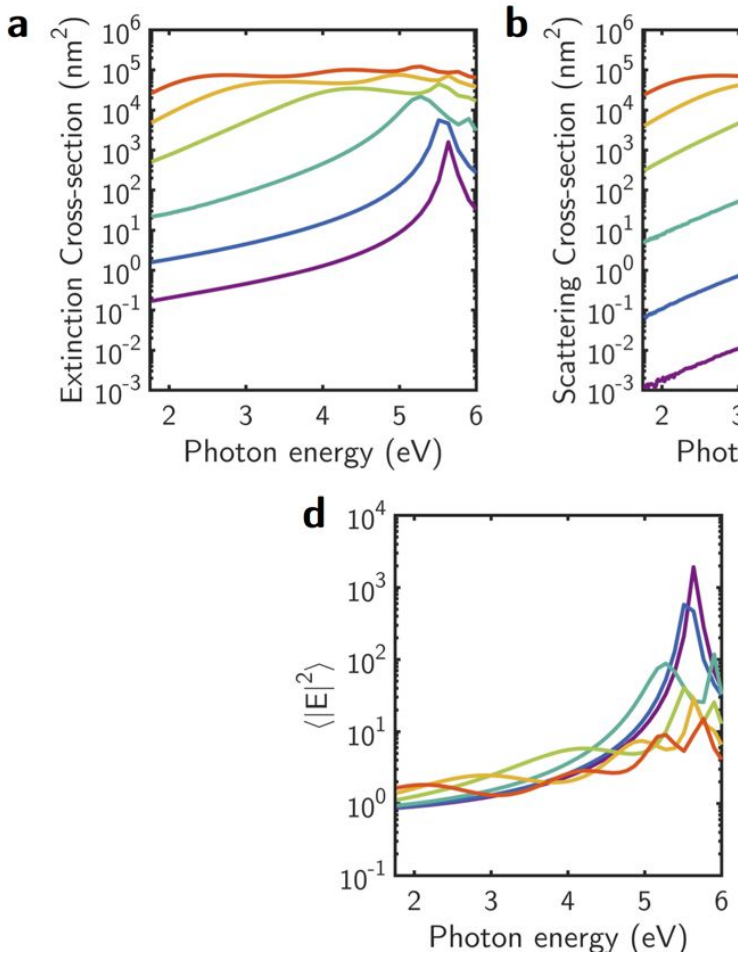

b
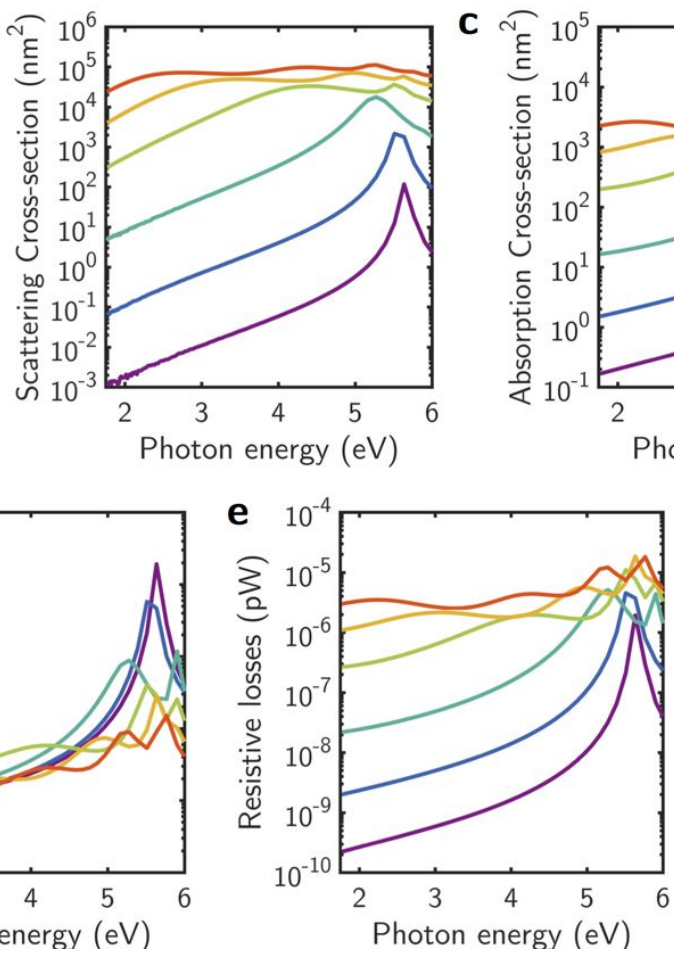

Figure S1. (a) Extinction, (b) scattering, (c) absorption cross-sections, (d) near-field enhancement averaged over the NP's surface and (e) resistive losses on Mg spheres of different radius: $5 \mathrm{~nm}$ (purple line), $10 \mathrm{~nm}$ (dark blue line), $20 \mathrm{~nm}$ (light blue line), $40 \mathrm{~nm}$ (green line), $60 \mathrm{~nm}$ (yellow line) and $80 \mathrm{~nm}$ (red line). 


\section{Group IV A materials}

- $\mathrm{Ti}$
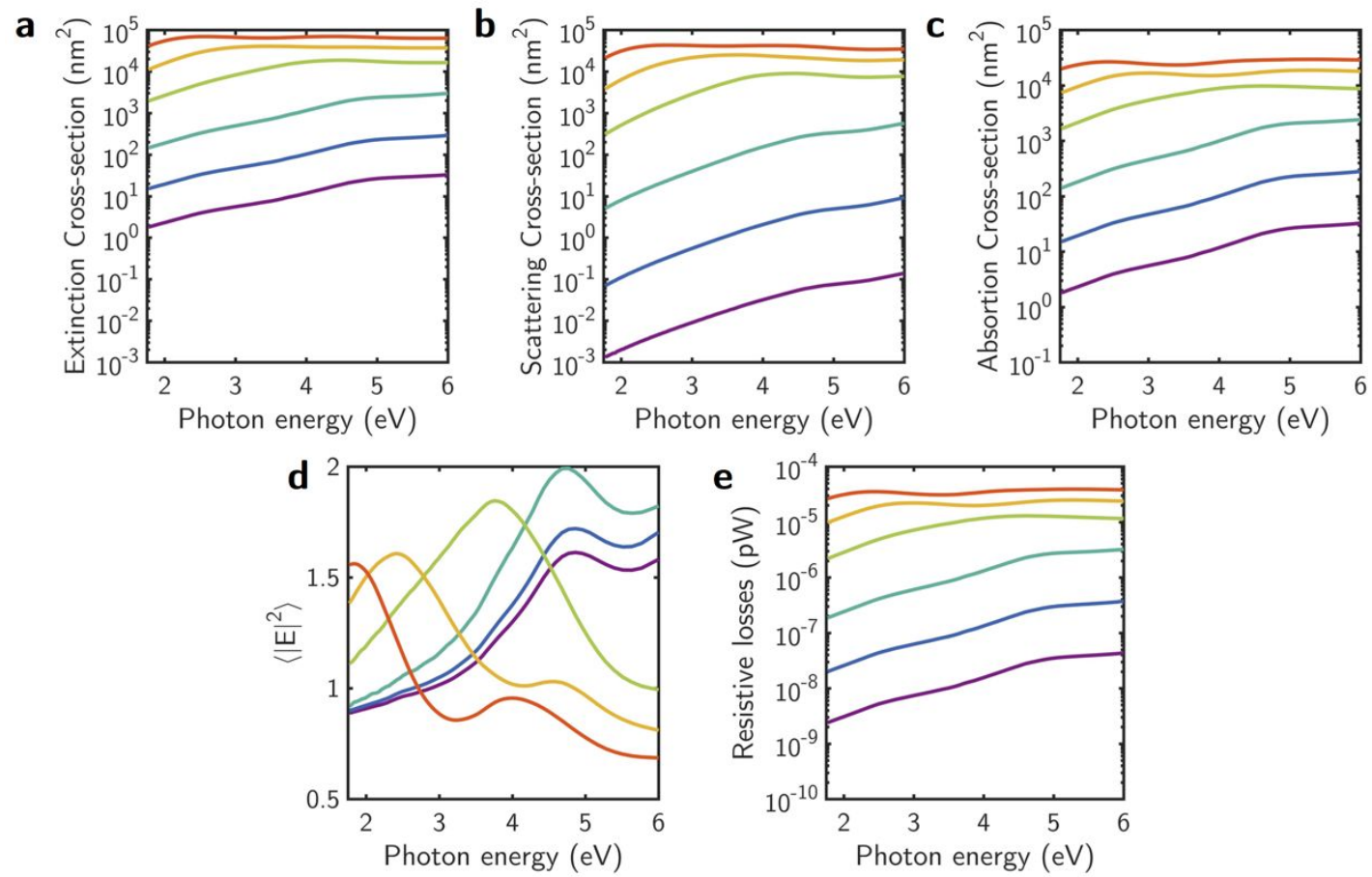

Figure S2. (a) Extinction, (b) scattering, (c) absorption cross-sections, (d) near-field enhancement averaged over the NP's surface and (e) resistive losses on Ti spheres of different radius: $5 \mathrm{~nm}$ (purple line), $10 \mathrm{~nm}$ (dark blue line), $20 \mathrm{~nm}$ (light blue line), $40 \mathrm{~nm}$ (green line), $60 \mathrm{~nm}$ (yellow line) and $80 \mathrm{~nm}$ (red line).

\section{Group VI A materials}

- $\mathrm{Cr}$ 

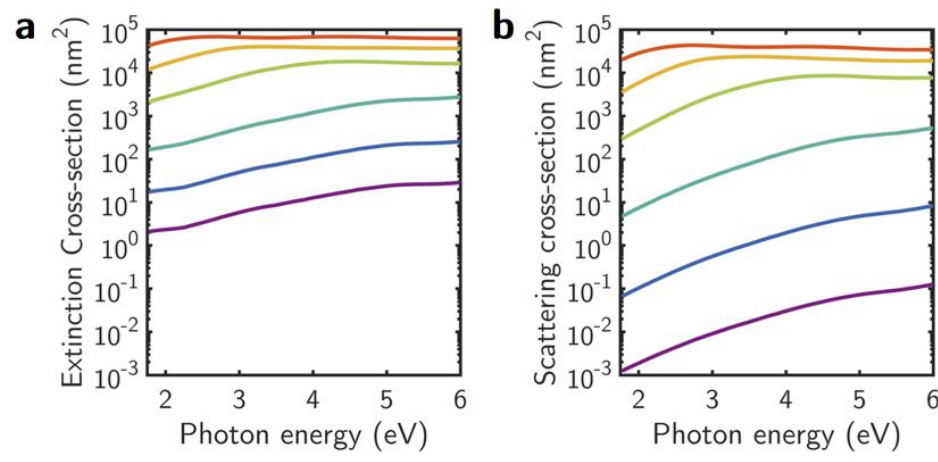

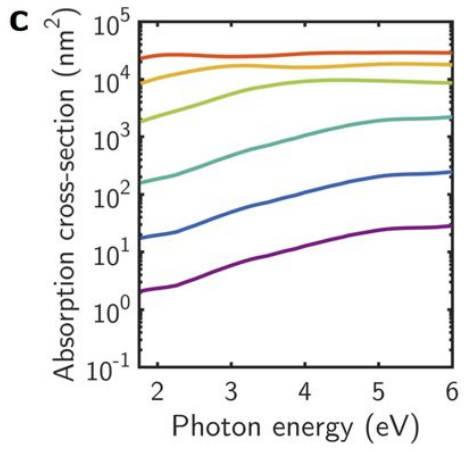

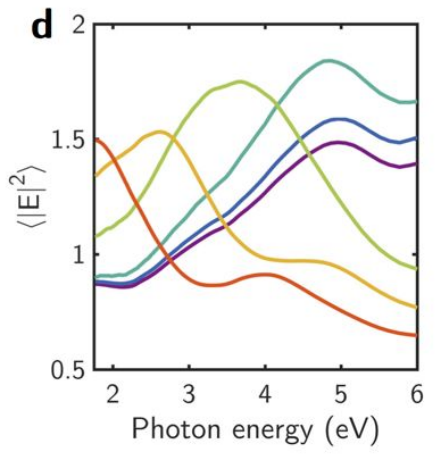

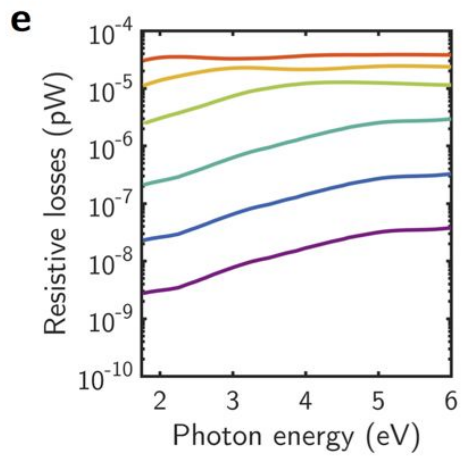

Figure S3. (a) Extinction, (b) scattering, (c) absorption cross-sections, (d) near-field enhancement averaged over the NP's surface and (e) resistive losses on $\mathrm{Cr}$ spheres of different radius: $5 \mathrm{~nm}$ (purple line), $10 \mathrm{~nm}$ (dark blue line), $20 \mathrm{~nm}$ (light blue line), $40 \mathrm{~nm}$ (green line), $60 \mathrm{~nm}$ (yellow line) and $80 \mathrm{~nm}$ (red line).

- Mo
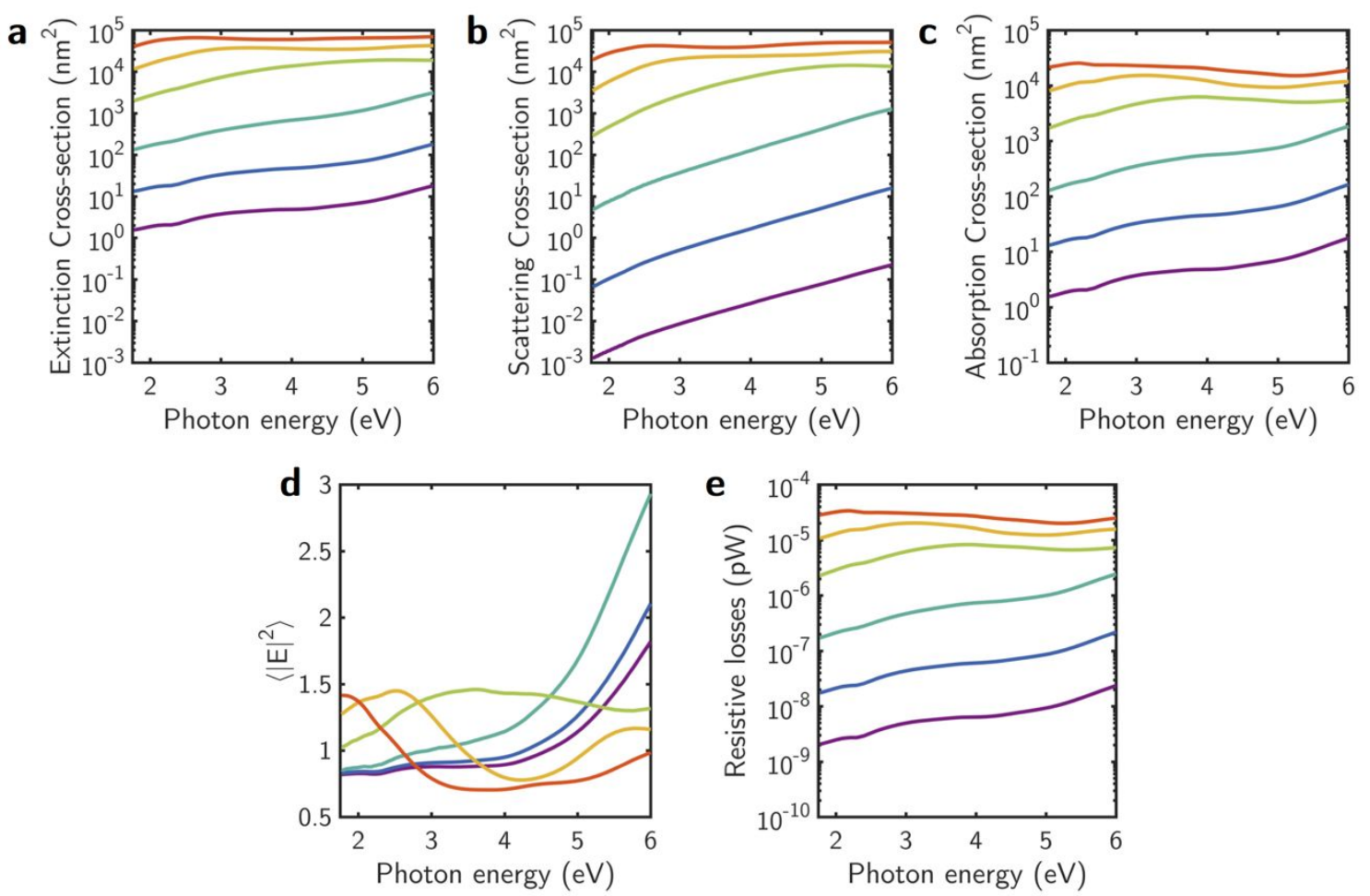

Figure S4. (a) Extinction, (b) scattering, (c) absorption cross-sections, (d) near-field enhancement averaged over the NP's surface and (e) resistive losses on Mo spheres of different radius: $5 \mathrm{~nm}$ (purple line), $10 \mathrm{~nm}$ (dark blue line), $20 \mathrm{~nm}$ (light blue line), $40 \mathrm{~nm}$ (green line), $60 \mathrm{~nm}$ (yellow line) and $80 \mathrm{~nm}$ (red line). 

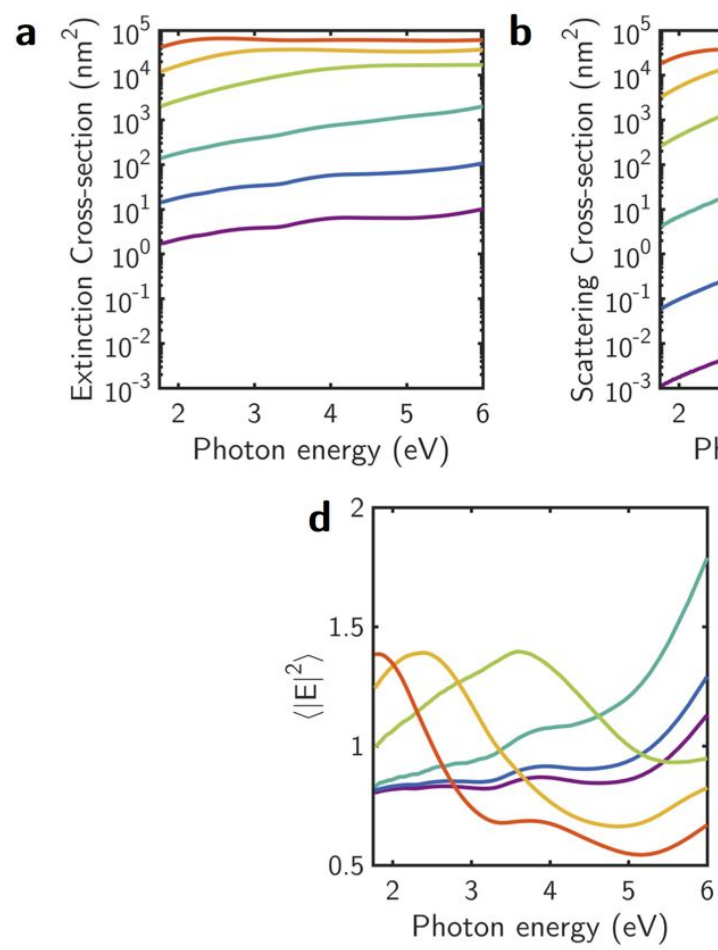
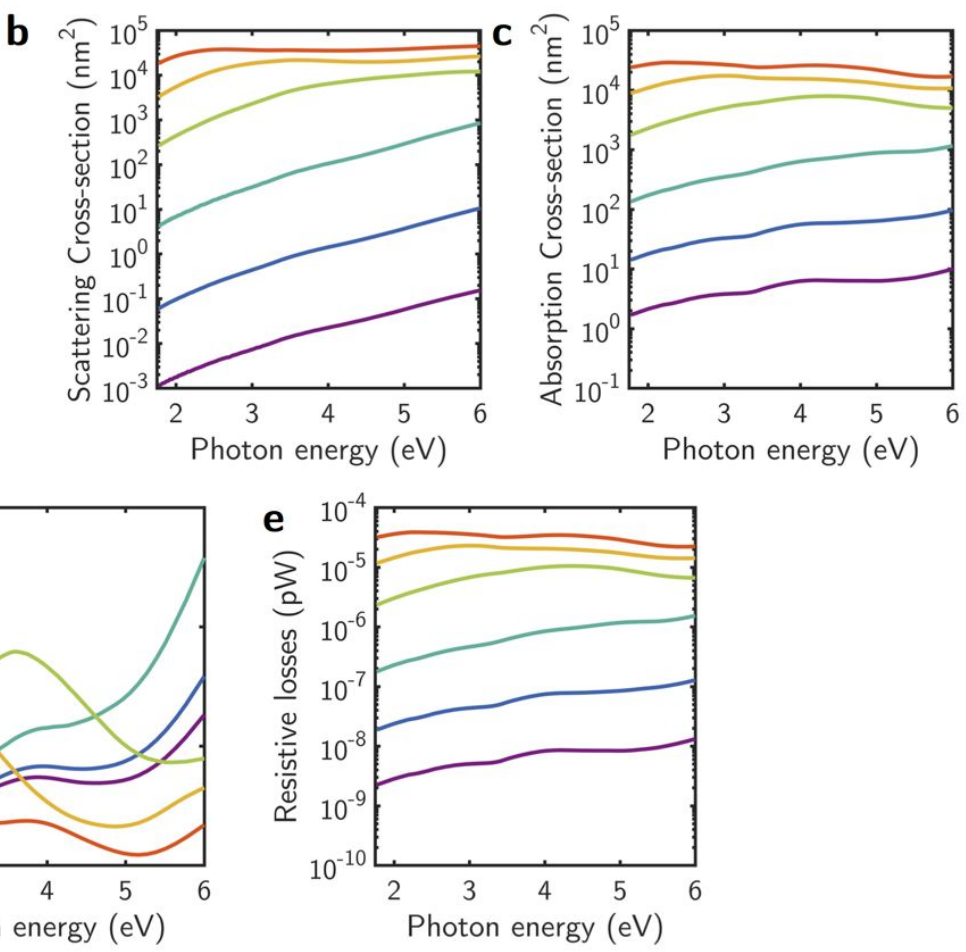

Figure S5. (a) Extinction, (b) scattering, (c) absorption cross-sections, (d) near-field enhancement averaged over the NP's surface and (e) resistive losses on W spheres of different radius: $5 \mathrm{~nm}$ (purple line), $10 \mathrm{~nm}$ (dark blue line), $20 \mathrm{~nm}$ (light blue line), $40 \mathrm{~nm}$ (green line), $60 \mathrm{~nm}$ (yellow line) and $80 \mathrm{~nm}$ (red line).

\section{Group VII A materials}

- $\operatorname{Re}$
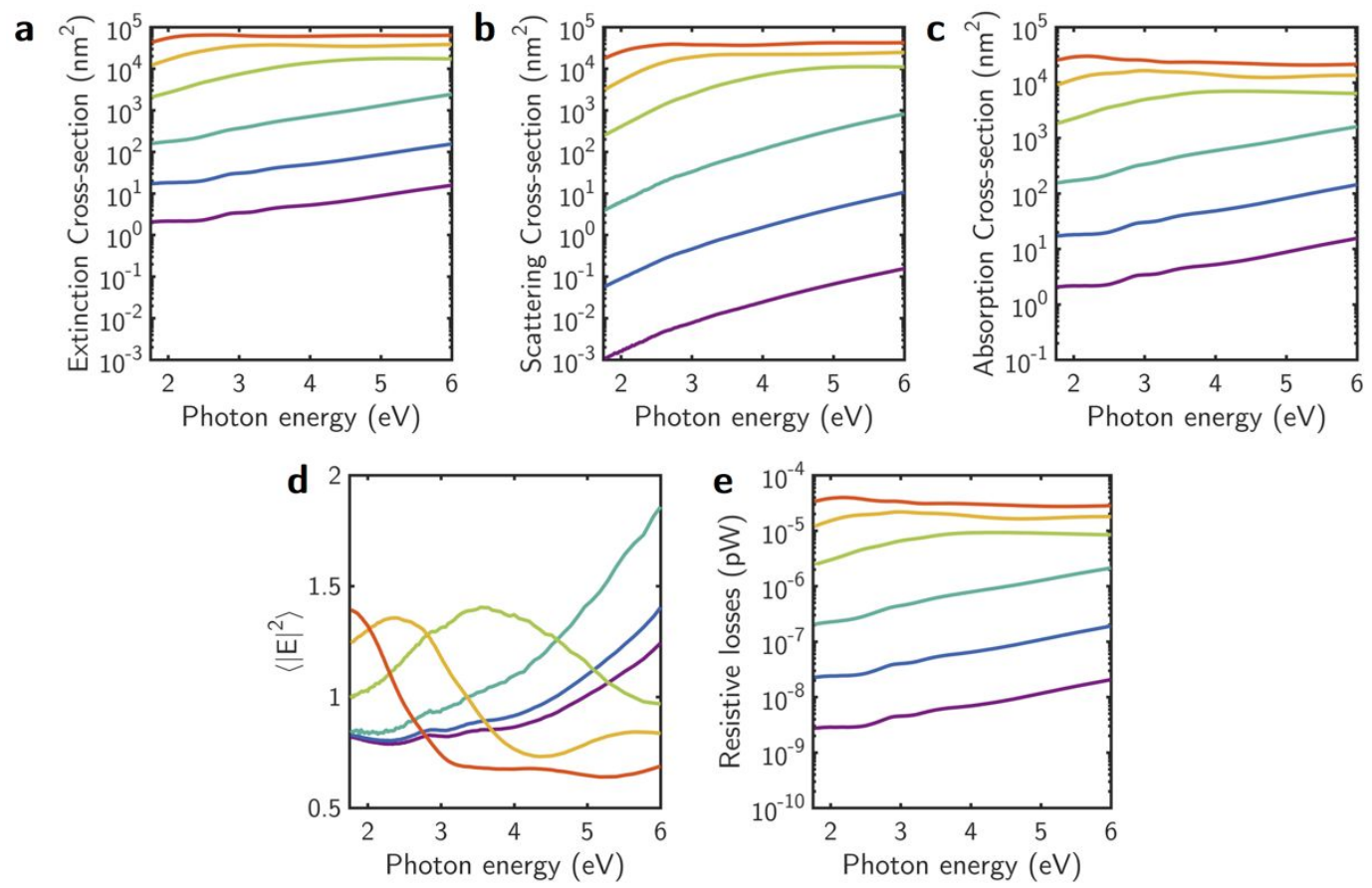
Figure S6. (a) Extinction, (b) scattering, (c) absorption cross-sections, (d) near-field enhancement averaged over the NP's surface and (e) resistive losses on Re spheres of different radius: $5 \mathrm{~nm}$ (purple line), $10 \mathrm{~nm}$ (dark blue line), $20 \mathrm{~nm}$ (light blue line), $40 \mathrm{~nm}$ (green line), $60 \mathrm{~nm}$ (yellow line) and $80 \mathrm{~nm}$ (red line).

\section{Group VIII A materials}

- $\mathrm{Fe}$
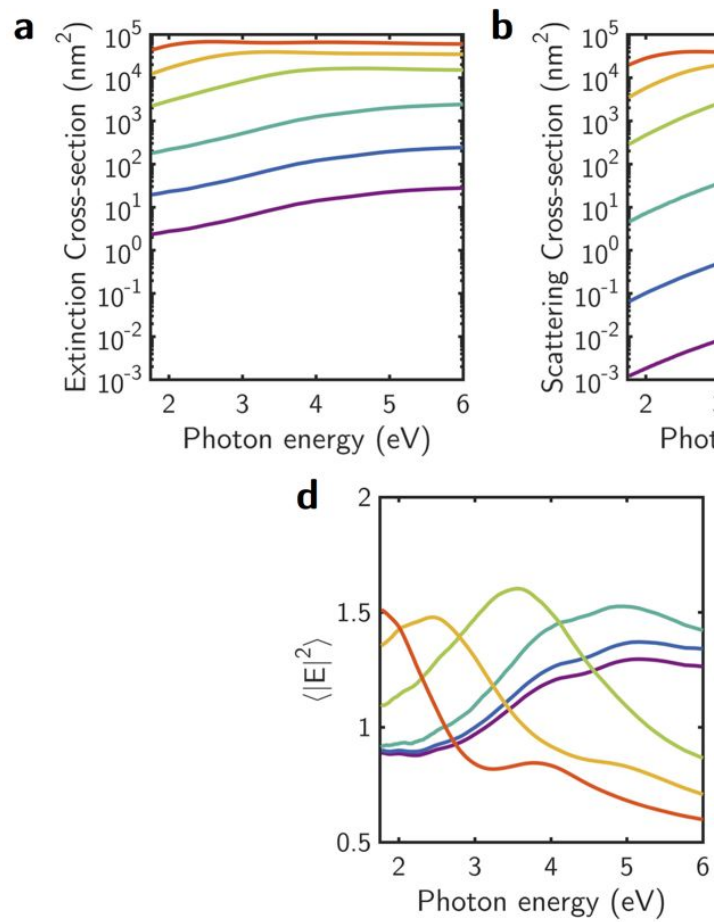
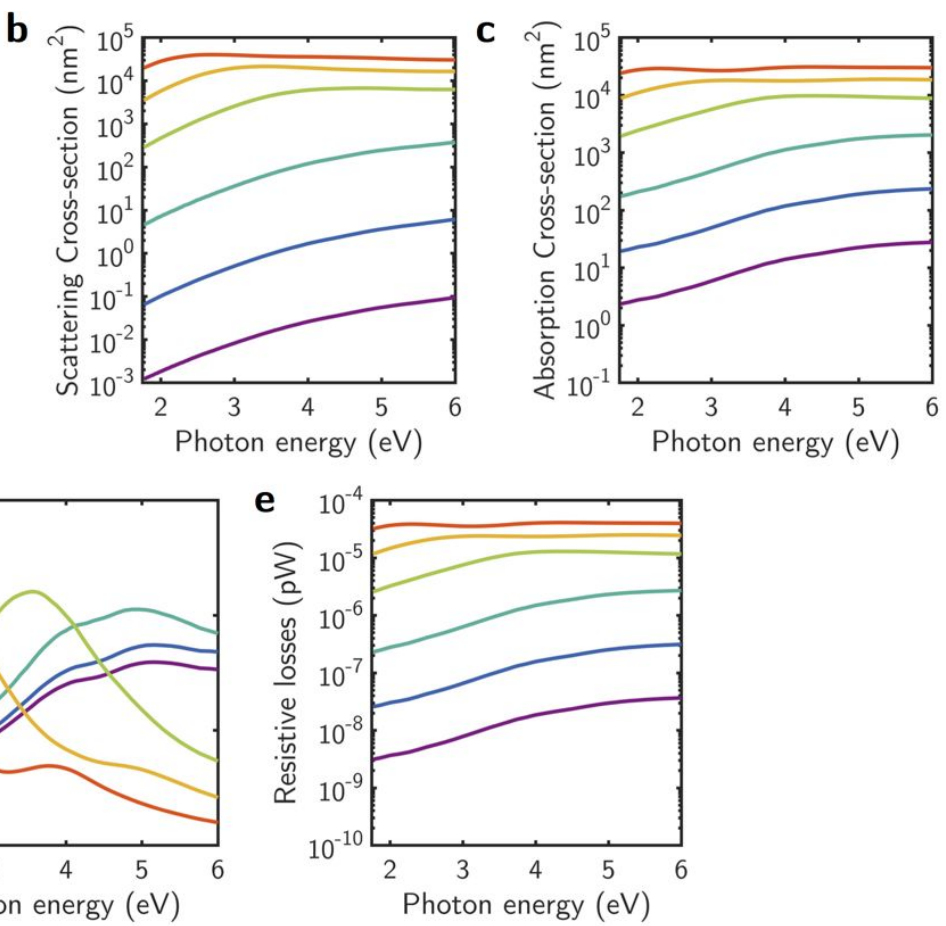

Figure S7. (a) Extinction, (b) scattering, (c) absorption cross-sections, (d) near-field enhancement averaged over the NP's surface and (e) resistive losses on Fe spheres of different radius: $5 \mathrm{~nm}$ (purple line), $10 \mathrm{~nm}$ (dark blue line), $20 \mathrm{~nm}$ (light blue line), $40 \mathrm{~nm}$ (green line), $60 \mathrm{~nm}$ (yellow line) and $80 \mathrm{~nm}$ (red line).

\section{- $\mathrm{Ru}$}



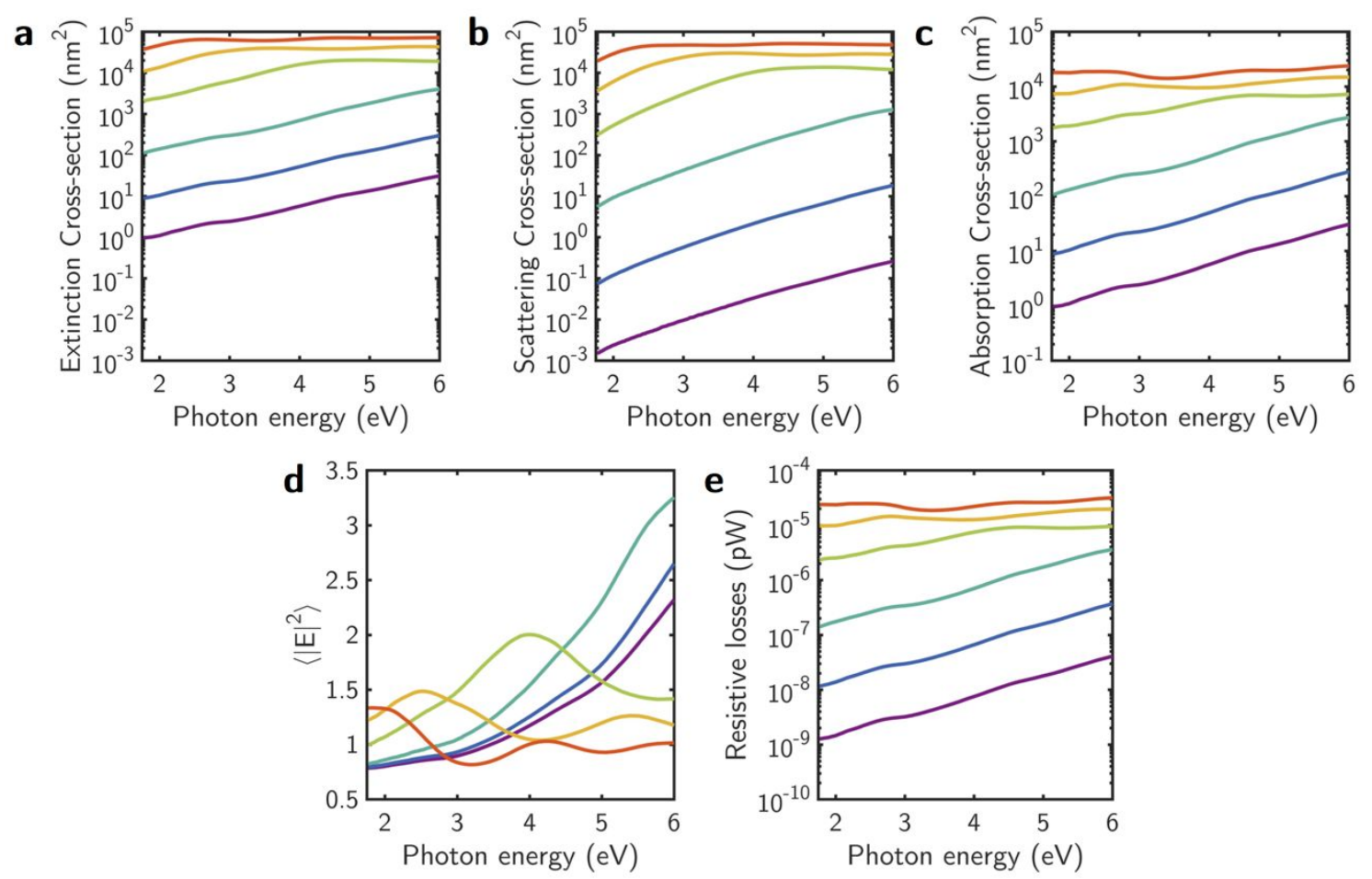

Figure S8. (a) Extinction, (b) scattering, (c) absorption cross-sections, (d) near-field enhancement averaged over the NP's surface and (e) resistive losses on Ru spheres of different radius: $5 \mathrm{~nm}$ (purple line), $10 \mathrm{~nm}$ (dark blue line), $20 \mathrm{~nm}$ (light blue line), $40 \mathrm{~nm}$ (green line), $60 \mathrm{~nm}$ (yellow line) and $80 \mathrm{~nm}$ (red line).

- Co
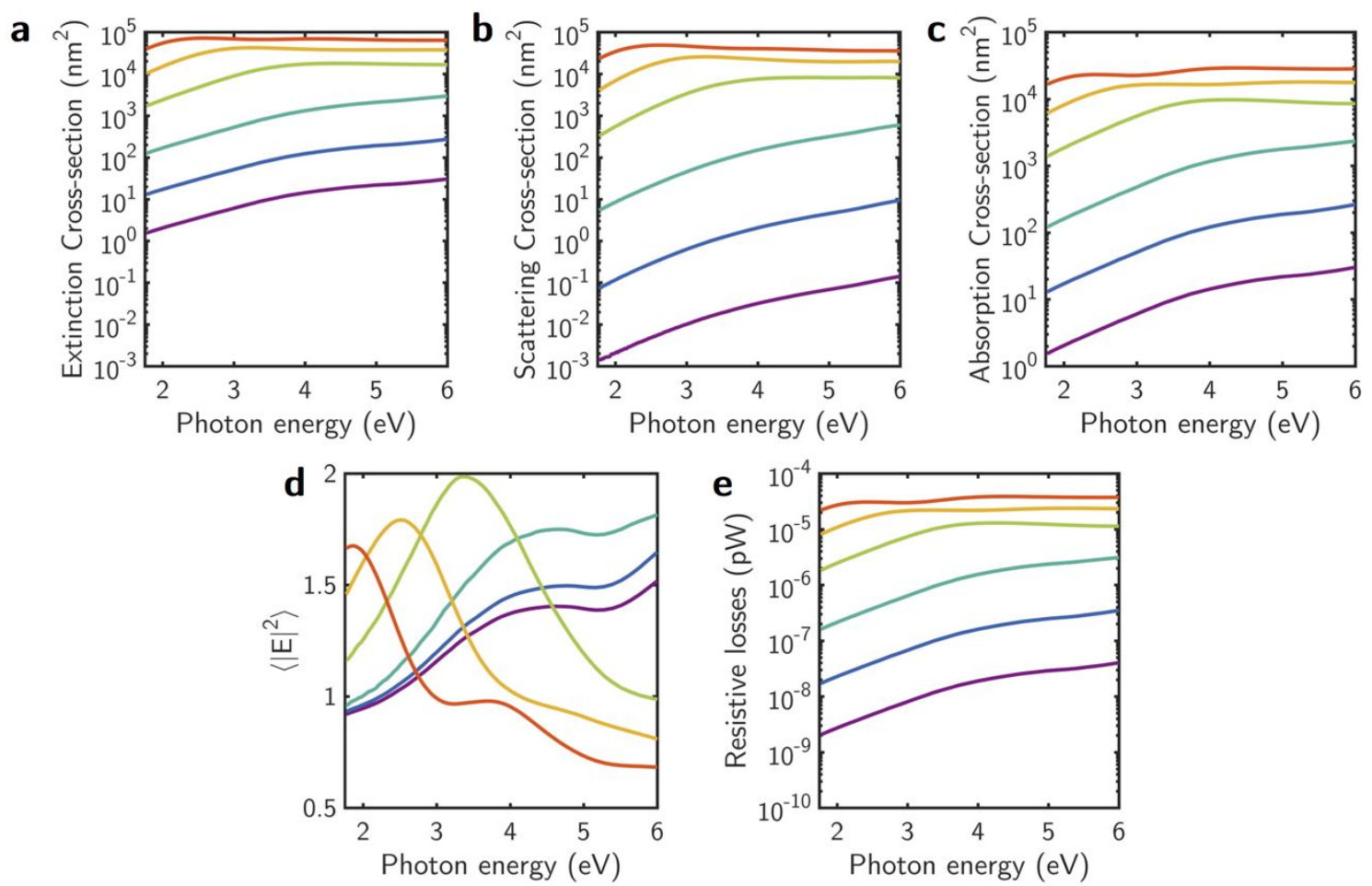

Figure S9. (a) Extinction, (b) scattering, (c) absorption cross-sections, (d) near-field enhancement averaged over the NP's surface and (e) resistive losses on Co spheres of different radius: $5 \mathrm{~nm}$ (purple line), $10 \mathrm{~nm}$ (dark blue line), $20 \mathrm{~nm}$ (light blue line), $40 \mathrm{~nm}$ (green line), $60 \mathrm{~nm}$ (yellow line) and $80 \mathrm{~nm}$ (red line). 
- $\mathrm{Rh}$
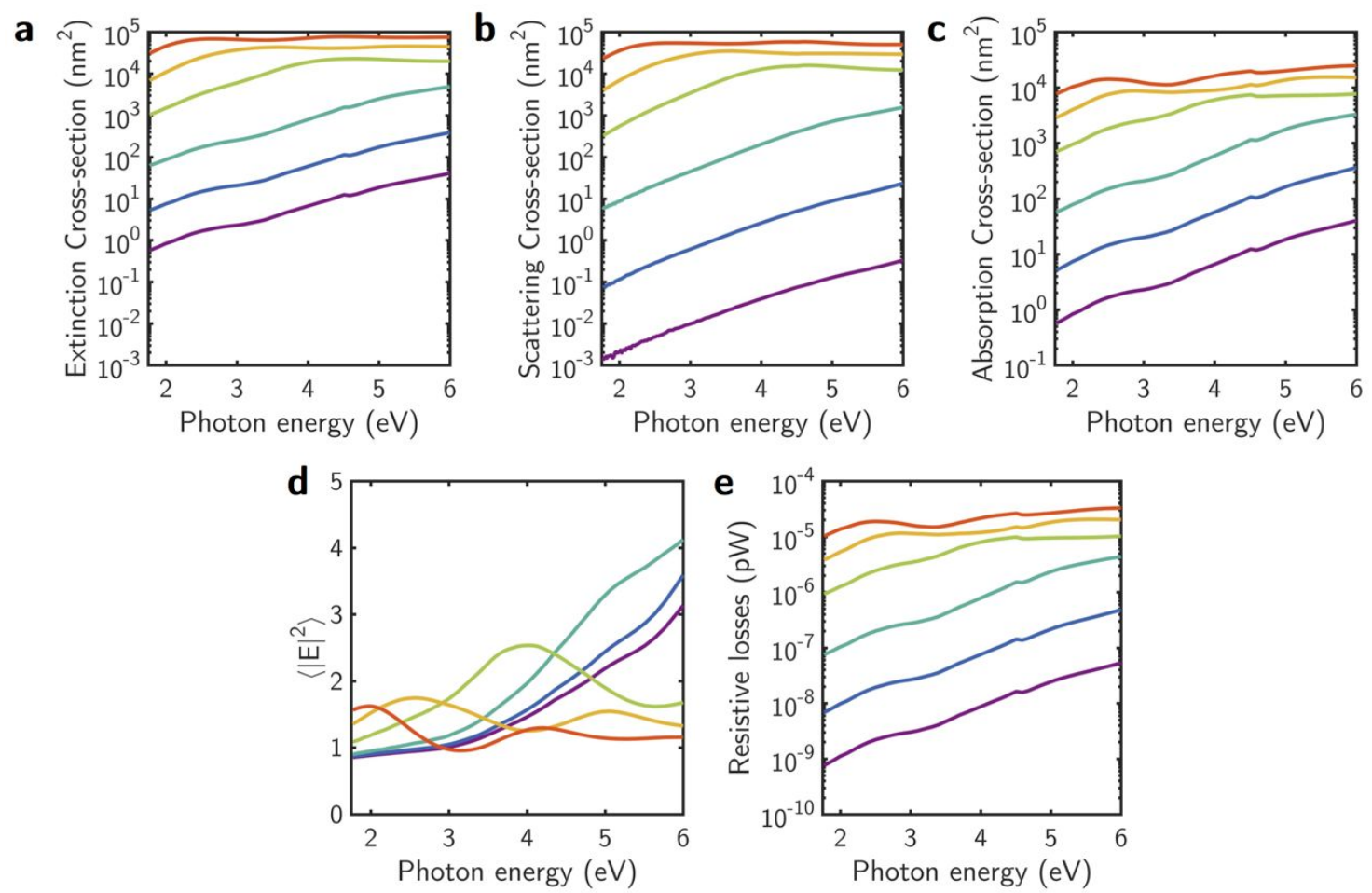

Figure S10. (a) Extinction, (b) scattering, (c) absorption cross-sections, (d) near-field enhancement averaged over the NP's surface and (e) resistive losses on Rh spheres of different radius: $5 \mathrm{~nm}$ (purple line), $10 \mathrm{~nm}$ (dark blue line), $20 \mathrm{~nm}$ (light blue line), $40 \mathrm{~nm}$ (green line), $60 \mathrm{~nm}$ (yellow line) and $80 \mathrm{~nm}$ (red line).

- $\mathrm{Ni}$
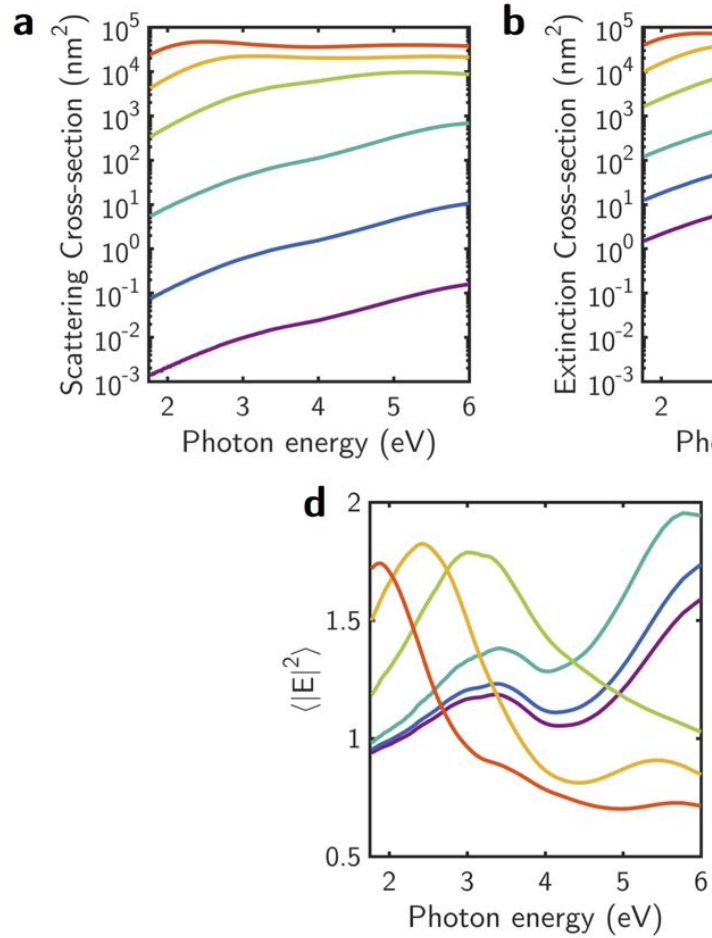

b

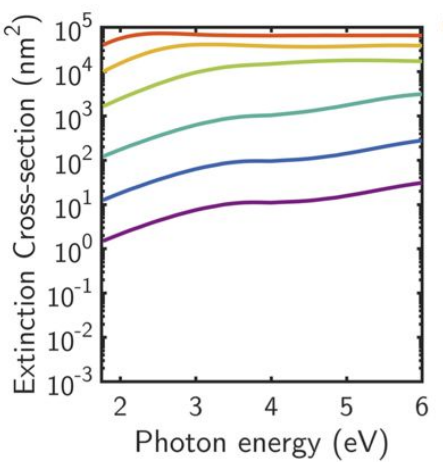

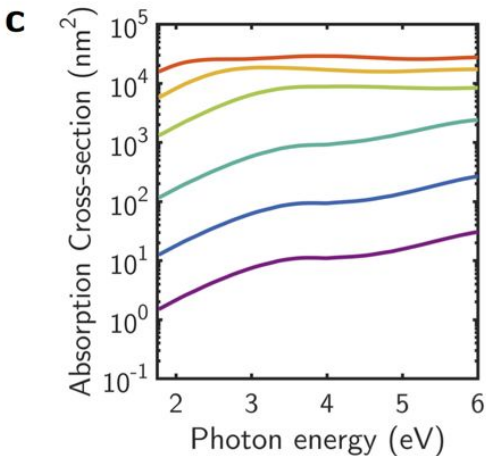

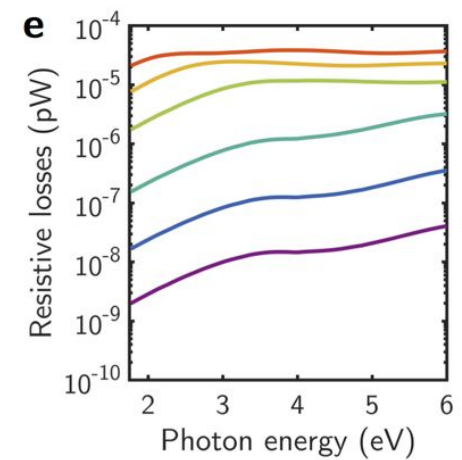


Figure S11. (a) Extinction, (b) scattering, (c) absorption cross-sections, (d) near-field enhancement averaged over the NP's surface and (e) resistive losses on Ni spheres of different radius: $5 \mathrm{~nm}$ (purple line), $10 \mathrm{~nm}$ (dark blue line), $20 \mathrm{~nm}$ (light blue line), $40 \mathrm{~nm}$ (green line), $60 \mathrm{~nm}$ (yellow line) and $80 \mathrm{~nm}$ (red line).

- Pd
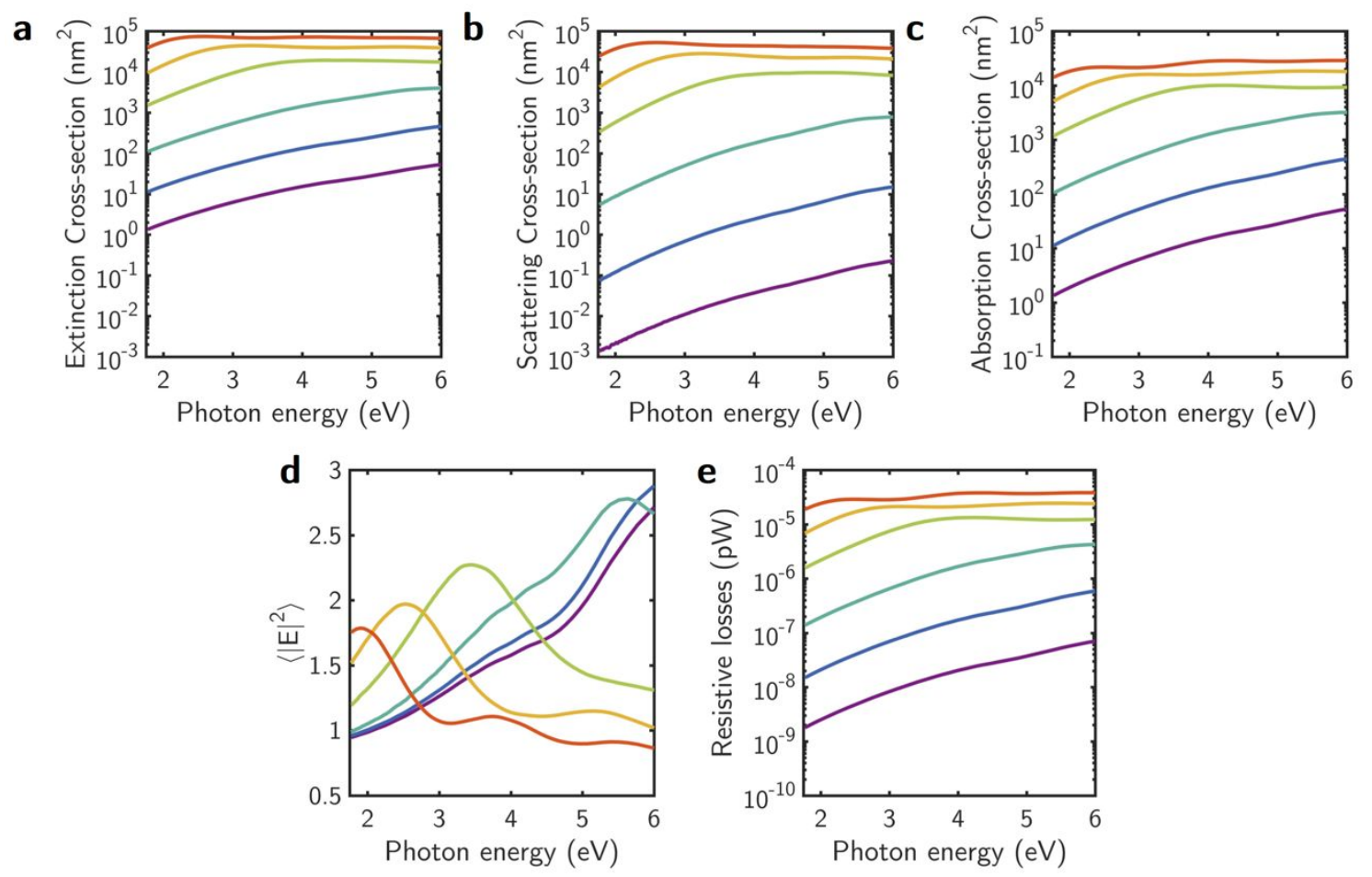

Figure S12. (a) Extinction, (b) scattering, (c) absorption cross-sections, (d) near-field enhancement averaged over the NP's surface and (e) resistive losses on Pd spheres of different radius: $5 \mathrm{~nm}$ (purple line), $10 \mathrm{~nm}$ (dark blue line), $20 \mathrm{~nm}$ (light blue line), $40 \mathrm{~nm}$ (green line), $60 \mathrm{~nm}$ (yellow line) and $80 \mathrm{~nm}$ (red line).

- $\mathrm{Pt}$ 

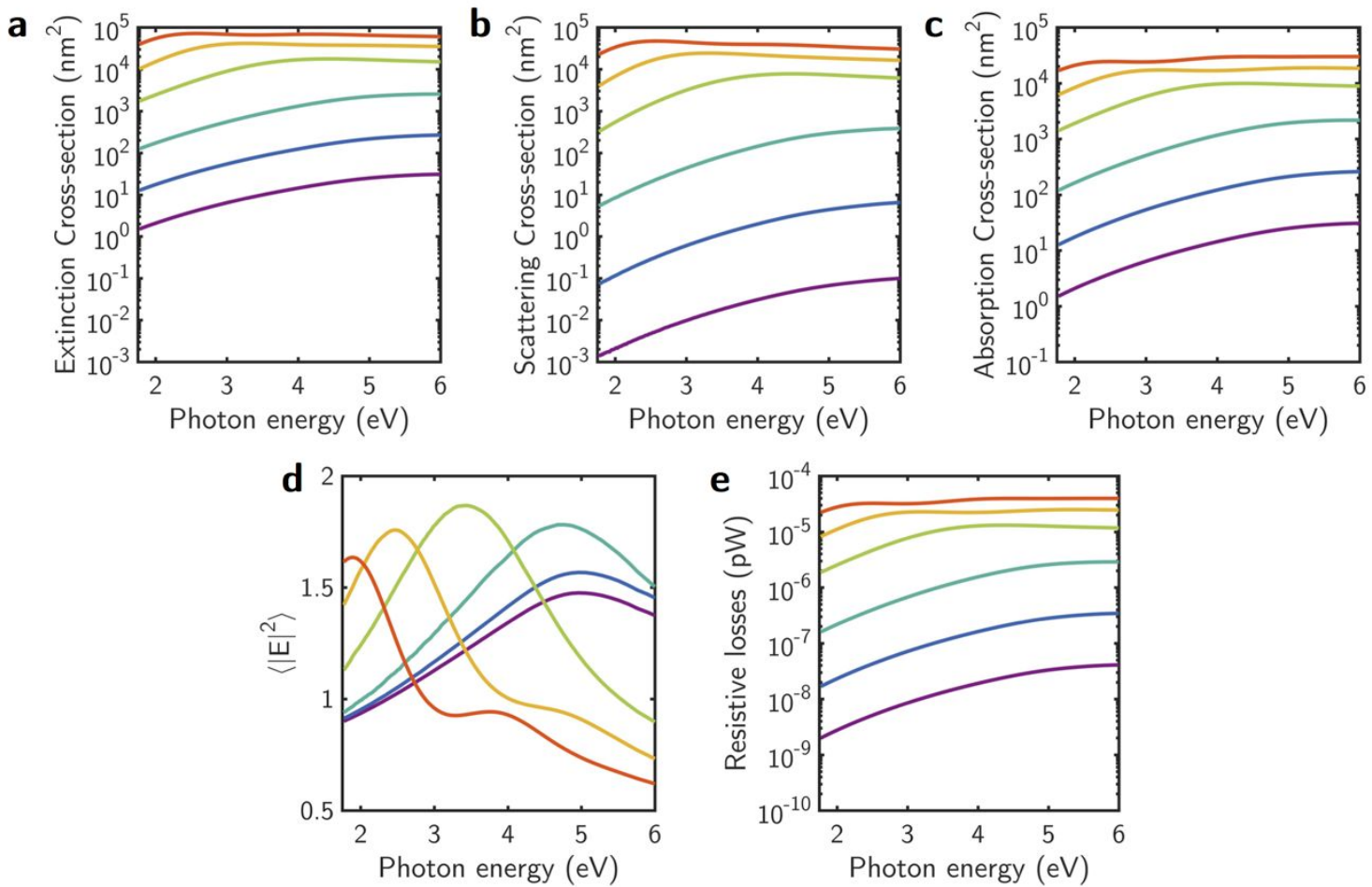

Figure S13. (a) Extinction, (b) scattering, (c) absorption cross-sections, (d) near-field enhancement averaged over the NP's surface and (e) resistive losses on Pt spheres of different radius: $5 \mathrm{~nm}$ (purple line), $10 \mathrm{~nm}$ (dark blue line), $20 \mathrm{~nm}$ (light blue line), $40 \mathrm{~nm}$ (green line), $60 \mathrm{~nm}$ (yellow line) and $80 \mathrm{~nm}$ (red line).

\section{Group II B materials}

- $\mathrm{Cu}$
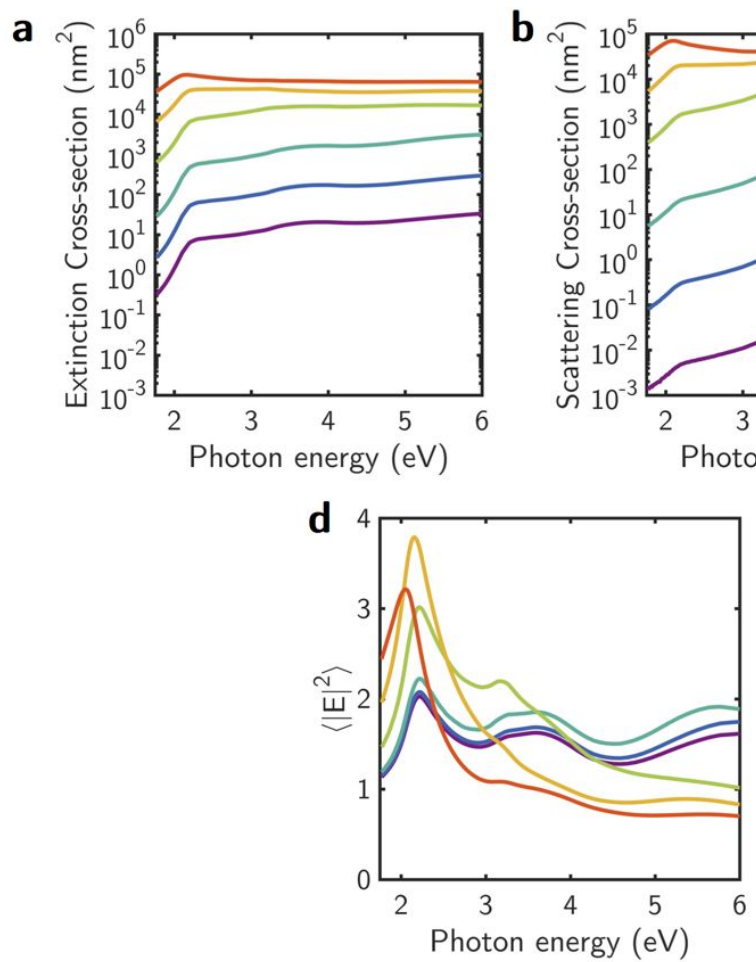
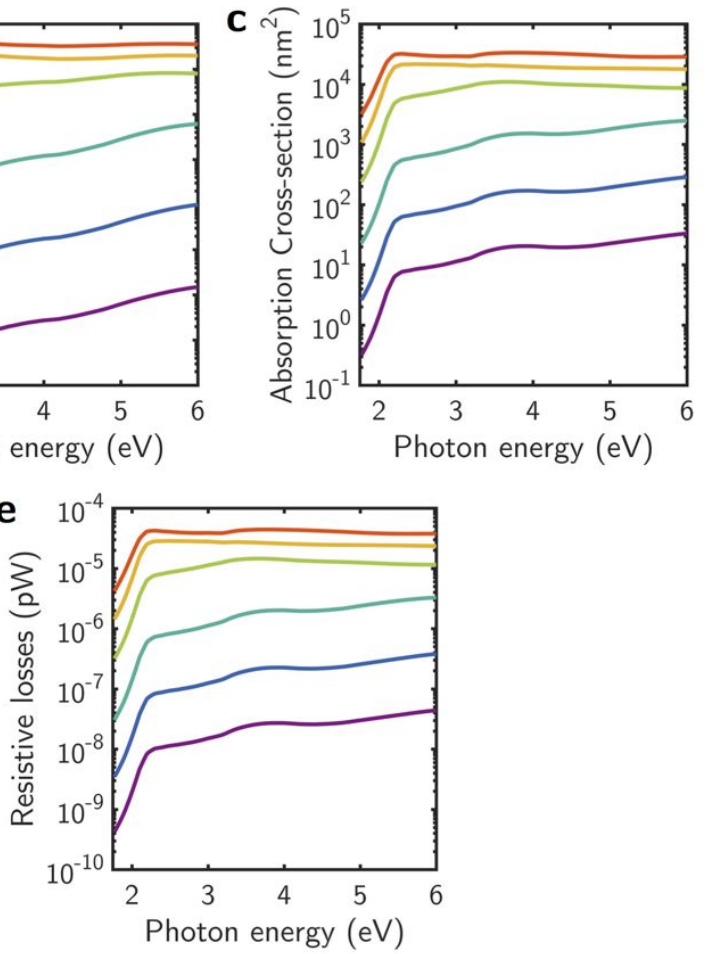
Figure S14. (a) Extinction, (b) scattering, (c) absorption cross-sections, (d) near-field enhancement averaged over the NP's surface and (e) resistive losses on Cu spheres of different radius: $5 \mathrm{~nm}$ (purple line), $10 \mathrm{~nm}$ (dark blue line), $20 \mathrm{~nm}$ (light blue line), $40 \mathrm{~nm}$ (green line), $60 \mathrm{~nm}$ (yellow line) and $80 \mathrm{~nm}$ (red line).

- $\mathrm{Ag}$

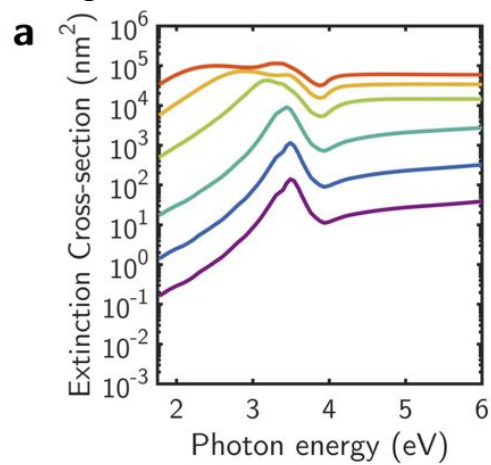

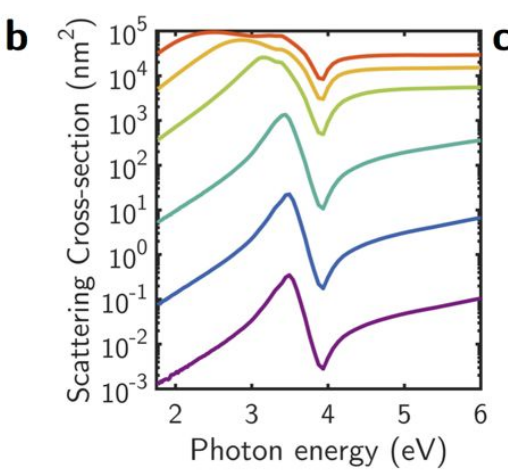

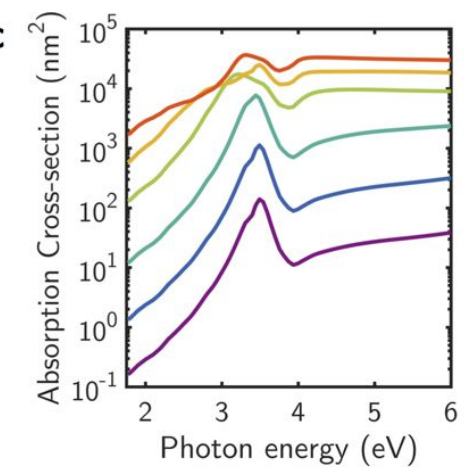

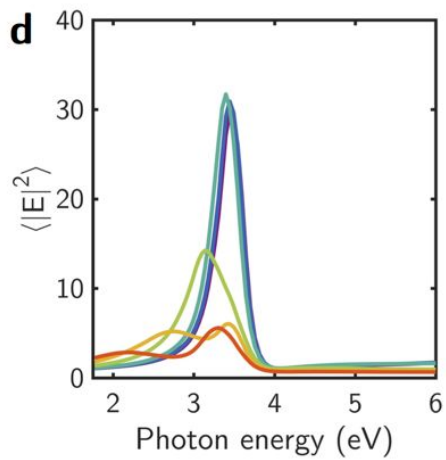

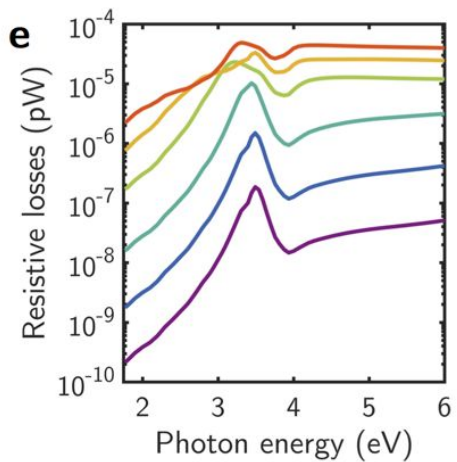

Figure S15. (a) Extinction, (b) scattering, (c) absorption cross-sections, (d) near-field enhancement averaged over the NP's surface and (e) resistive losses on Ag spheres of different radius: $5 \mathrm{~nm}$ (purple line), $10 \mathrm{~nm}$ (dark blue line), $20 \mathrm{~nm}$ (light blue line), $40 \mathrm{~nm}$ (green line), $60 \mathrm{~nm}$ (yellow line) and $80 \mathrm{~nm}$ (red line).

- $\mathrm{Au}$ 
a

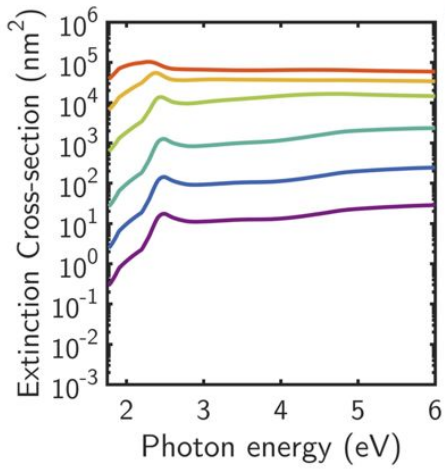

b

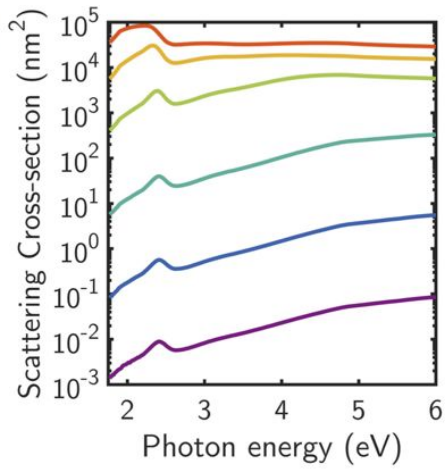

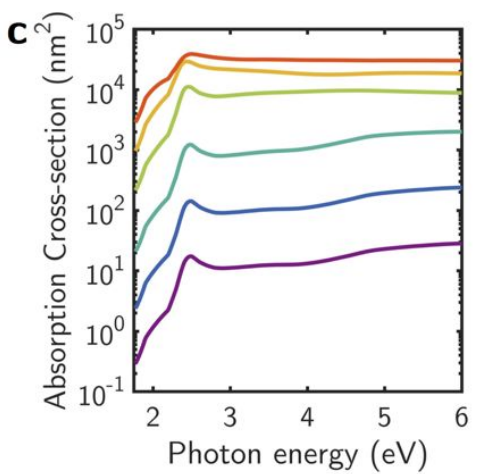
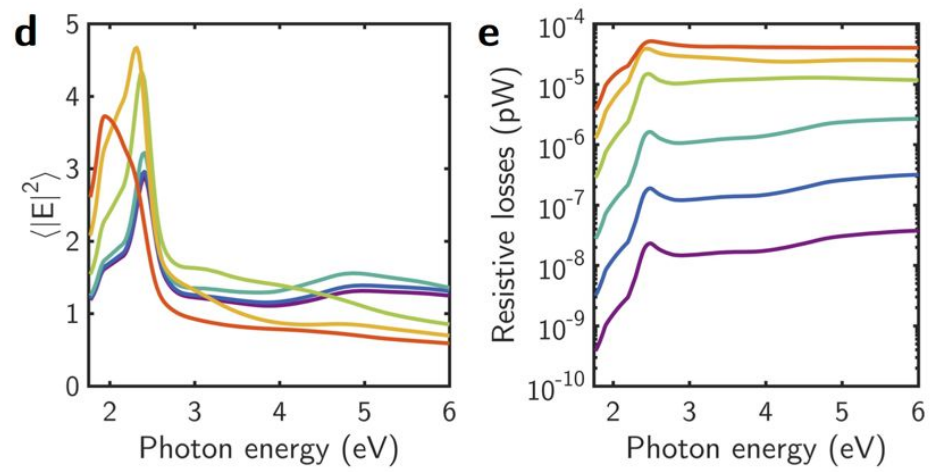

Figure S16. (a) Extinction, (b) scattering, (c) absorption cross-sections, (d) near-field enhancement averaged over the NP's surface and (e) resistive losses on Au spheres of different radius: $5 \mathrm{~nm}$ (purple line), $10 \mathrm{~nm}$ (dark blue line), $20 \mathrm{~nm}$ (light blue line), $40 \mathrm{~nm}$ (green line), $60 \mathrm{~nm}$ (yellow line) and $80 \mathrm{~nm}$ (red line).

\section{Group III B materials}

- Al
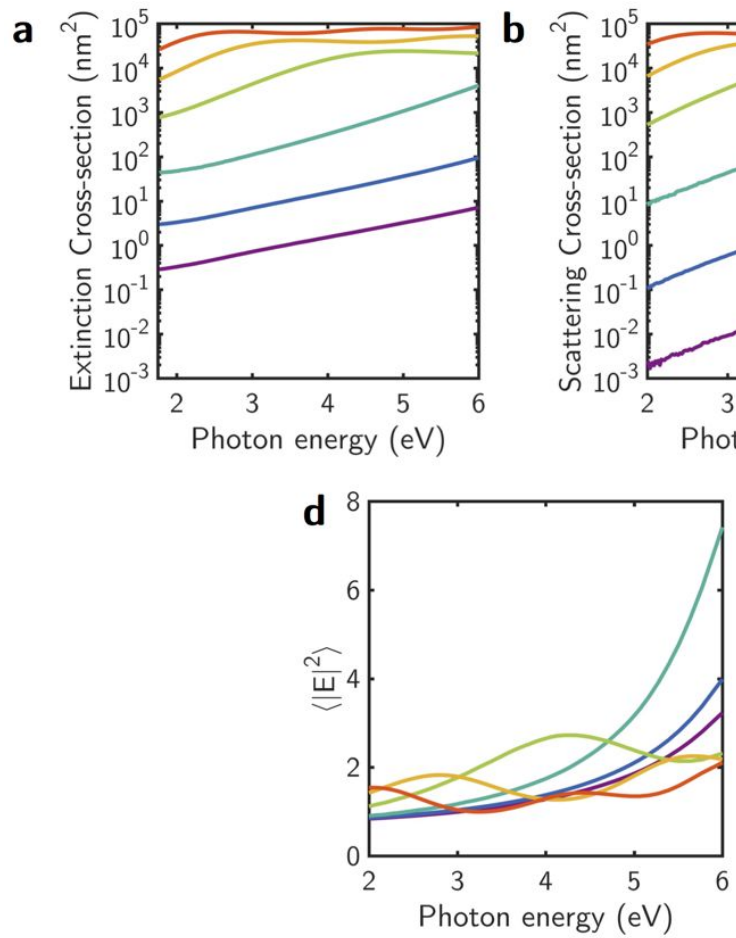
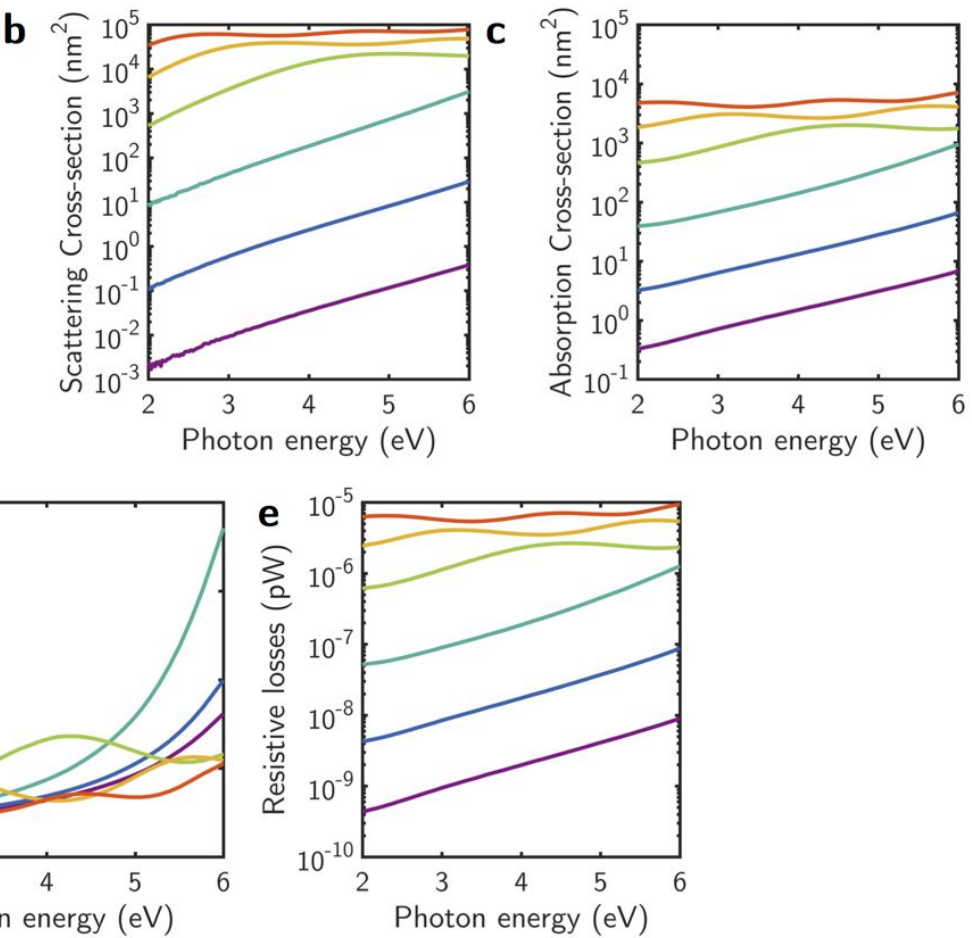

Figure S17. (a) Extinction, (b) scattering, (c) absorption cross-sections, (d) near-field enhancement averaged over the NP's surface and (e) resistive losses on Ga spheres of different radius: $5 \mathrm{~nm}$ (purple line), $10 \mathrm{~nm}$ (dark blue line), $20 \mathrm{~nm}$ (light blue line), $40 \mathrm{~nm}$ (green line), $60 \mathrm{~nm}$ (yellow line) and $80 \mathrm{~nm}$ (red line). 
- $\mathrm{Ga}$
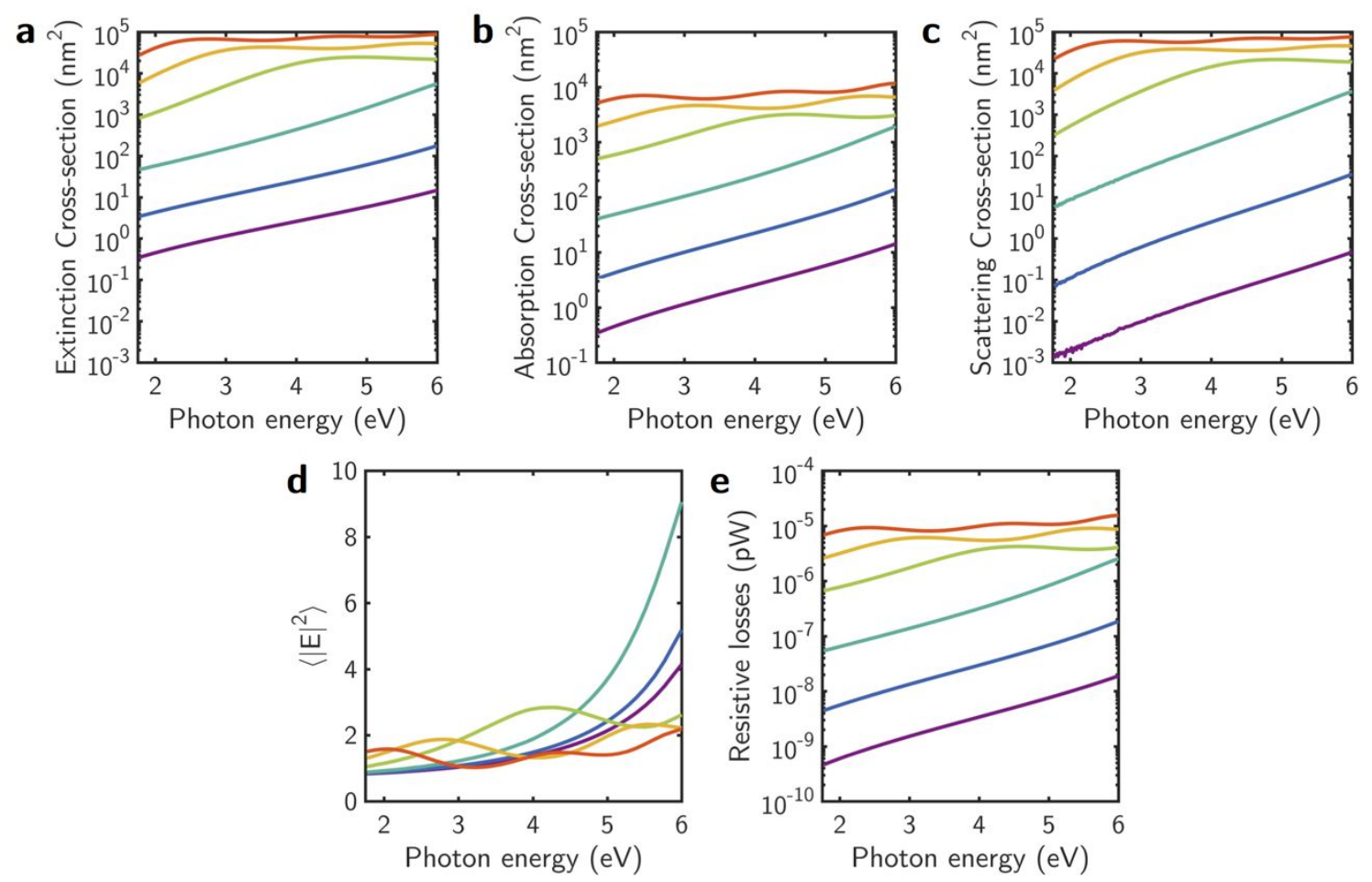

Figure S18. (a) Extinction, (b) scattering, (c) absorption cross-sections, (d) near-field enhancement averaged over the NP's surface and (e) resistive losses on Ga spheres of different radius: $5 \mathrm{~nm}$ (purple line), $10 \mathrm{~nm}$ (dark blue line), $20 \mathrm{~nm}$ (light blue line), $40 \mathrm{~nm}$ (green line), $60 \mathrm{~nm}$ (yellow line) and $80 \mathrm{~nm}$ (red line).

- In
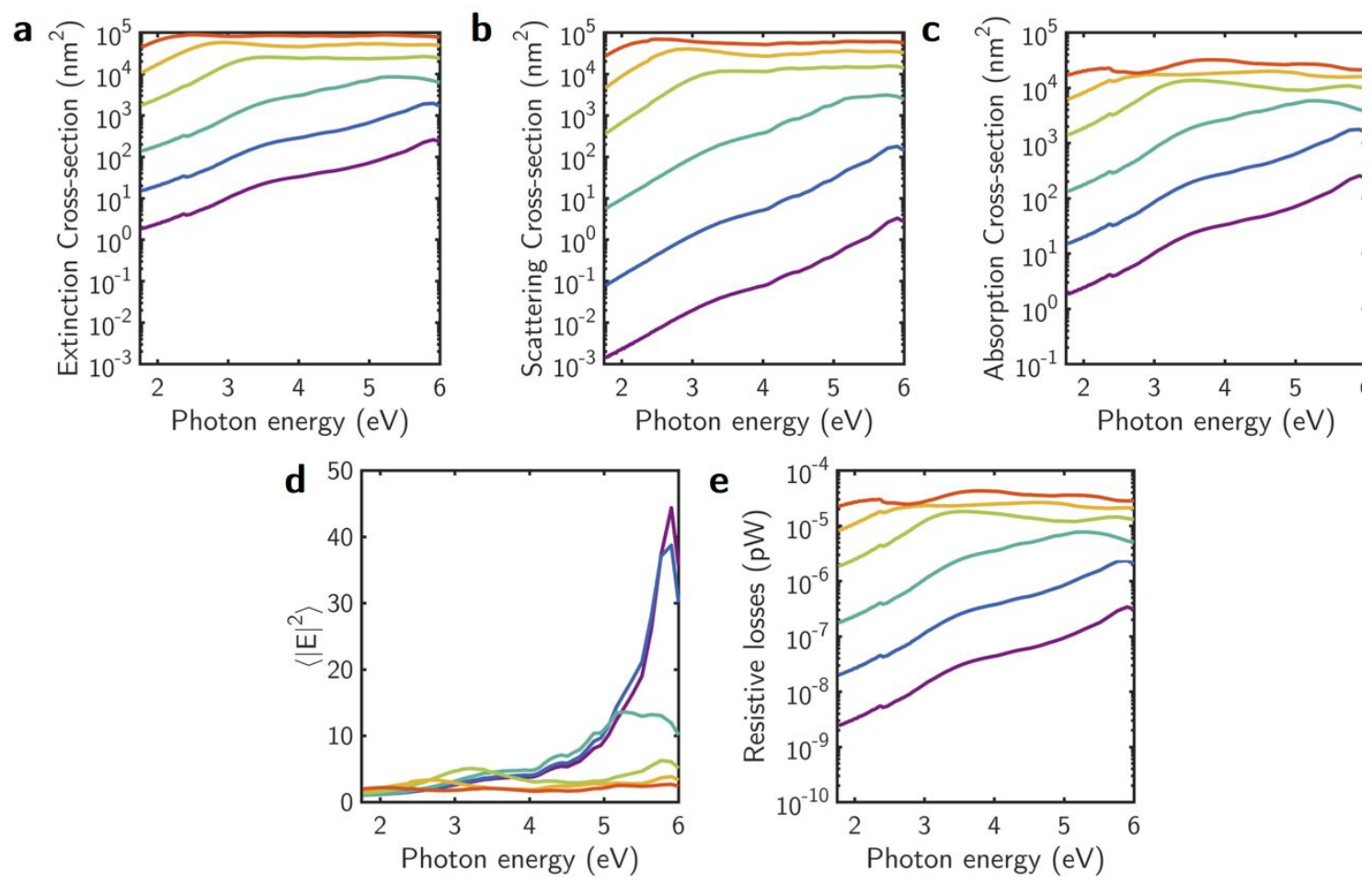
Figure S19. (a) Extinction, (b) scattering, (c) absorption cross-sections, (d) near-field enhancement averaged over the NP's surface and (e) resistive losses on In spheres of different radius: $5 \mathrm{~nm}$ (purple line), $10 \mathrm{~nm}$ (dark blue line), $20 \mathrm{~nm}$ (light blue line), $40 \mathrm{~nm}$ (green line), $60 \mathrm{~nm}$ (yellow line) and $80 \mathrm{~nm}$ (red line). 
Derivation of $d$ (atomic diameter) from data in Ref. [66]

The $d$ parameter (the diameter of the atomic constituents) can be directly derived from the tabulated value of the atomic volume per mole (V) in Ref. [66]. First we obtain the volume of each atom ( $\mathrm{V}$ ') by using the Avogadro number $\left(\mathrm{N}_{\mathrm{A}}=6.022 \cdot 10^{23} \mathrm{atoms} / \mathrm{mol}\right)$,

$$
V^{\prime}=V \frac{\mathrm{cm}^{3}}{\mathrm{~mol}} \cdot \frac{1 \mathrm{~mol}}{6.022 \cdot 10^{23} \text { atom }}
$$

Then, assuming that the atom is spherical, the value of $d$ is obtained directly obtained as:

$$
d=2\left(\frac{3 V^{\prime}}{4 \pi}\right)^{\frac{1}{3}}
$$

